Volume 7, No.2, April - June 2018

International Journal of Computing, Communications and Networking

Available Online at http://www.warse.org/ijcen/static/pdf/file/ijcen37722018.pdf

https://doi.org/10.30534/ijccn/2018/37722018

\title{
PROXY-ASSISTED CONTENT SHARING USING CONTENT CENTRIC NETWORKING (CCN) FOR RESOURCE-LIMITED MOBILE CONSUMER DEVICES.
}

\author{
Prof. Prasanna Kumar', Anjali Singh ${ }^{2}$, Anusha $\mathbf{M}^{3}$, Asha $K^{4}$, Mishra Maanshi ${ }^{5}$ \\ ${ }^{1}$ EWIT,India,prasannakumar@ewit.edu \\ ${ }^{2}$ EWIT, India, as0161947@gmail.com \\ ${ }^{3}$ EWIT, India, anuyaji1996@gmail.com \\ ${ }^{4}$ EWIT, India, asha.kv0506@gmail.com \\ ${ }^{5}$ EWIT, India, maanshimishra@gmail.com
}

\begin{abstract}
Named data networking (NDN) resolves the traditional TCP/IP based Internet problems (i.e., location dependent, complex usage, scalability, poor resource utilization etc.) and is considered as an eligible candidate for futuristic Internet paradigm. In NDN-based mobile ad hoc networks (MANETs), the participating nodes are operated in highly dynamic and challenge able environment such as low battery power, channel fluctuations, intermittent connectivity and so on. Due to the broadcast nature of the wireless channel, the NDN-based MANETs highlight severe issues (e.g., packet collisions, flooding, data redundancy, packet retransmissions), which further degrade the network performance. In this paper, to cope with these problems, we have proposed a novel protocol, named location-aware on-demand multipath caching and forwarding (LOMCF) for NDN-based MANETs. Performance of the proposed protocol is evaluated by using simulator, called ndnSIM. Extensive experiments along their results show that proposed protocol performs better as comparing to the other recent proposed protocols in terms of content retrieval time, Interest retransmissions, and the total number of Interest packets injected as well as discarded in the network.
\end{abstract}

Keywords - Content, Interest, Named Data

Networking, Information Centric, MANETs, Caching

\section{INTRODUCTION}

Currently, Internet architecture is based on the TCP/IP paradigm in which communication is held using a device or machine physical address. It is hard to update the current Internet architecture and further enhance its communication parameters, which is also called ossification of the Internet [1].

Furthermore, the TCP/IP-based Internet has several drawbacks (e.g., location dependent, security, inefficient resource utilization). Currently, the quantity of wireless devices (e.g., smart-phones, laptops, tabs) has increased sharply due to the rapid development in technology.

Fig. 1 shows the hourglass architecture of both NDN- (right side) and TCP/IP (left side)-based paradigms.

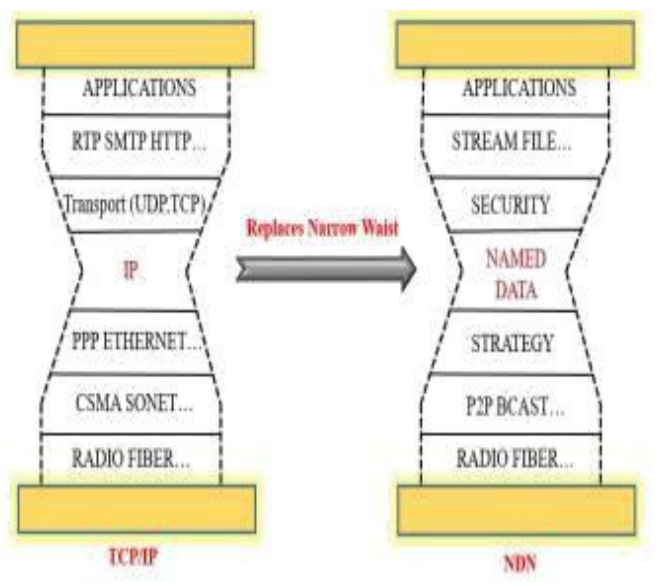

Fig. 1: NDN hourglass 
A mobile ad hoc network (MANET) is a combination of self-organized, resourceconstrained digital devices that can di-rectly communicate with each other using a short transmission range. In this network, the topology and interconnectivity are unpredictable, as nodes randomly move as well as dynamically appear and disappear from the network, as shown in Fig.2.Basically,a major type of communication in MANET is data-centric in nature, such as situation awareness information, software updates, surveillance data, and so on . For more than a decade, traditional IP-based protocols have been pro-posed for MANETs that are host-centric in nature.

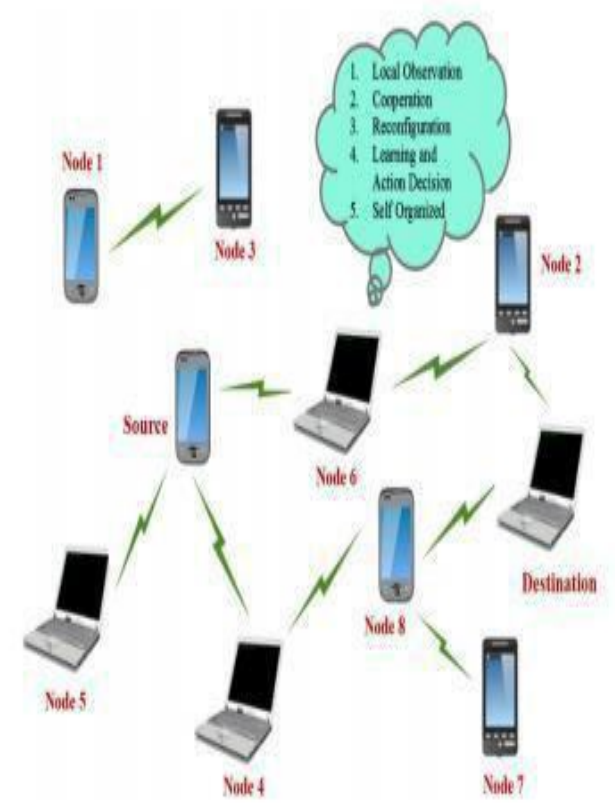

Fig. 2: Mobile ad hoc networks
Similar to wired networks, NDN also shows fruitful advantages in wireless ad hoc networks and cognitive radio ad hoc networks [28]-[29]. Due to the broad-cast nature of the wireless channel, the NDN-based MANETs highlight severe issues (e.g., packet collisions, flooding, data redundancy, packet retransmissions) that further degrades the network performance.

Previously, the nodes utilized the in-networking caching property of the NDN that can store data packets at any node (i.e., leave copy everywhere caching policy).Consequently, the data redundancy is also increased in the network. In this paper,we propose a novel protocol, location-aware on-

demand multipath caching and forwarding(LOMCF) for NDN-based MANETs.

\section{RELATED WORKS}

Previously, different opportunistic [24] and content-based[34] models were used to achieve similar goals as NDN.However, NDN has gained considerable popularity in the wireless arena due to its simple communication paradigm. The authors in [35] present an NDN-based video streaming approach in wireless ad hoc networks. The authors claim that the NDN approach shows efficient quality of service (QoS) as well as productive results in a resource-constrained MANET environment. Raaid et al., in [36], compare the performance of both NDN-based and IP-based MANETs. Their study shows that NDN-based MANETs are considered a good candidate for emerging recovery application and natural disaster scenarios.

In [37], the authors describe a new scheme (i.e., ECHANET) for multihop wireless ad hoc networks. The E-CHANET scheme utilizes an extra data structure (e.g., provider table) during its communication process. Moreover, the E-

CHANET 
scheme is proposed only for low-mobility scenarios and does not take into account the remaining energy of the nodes. Fabioet al. present a novel packet forwarding scheme that is based on bloom filters [38].

In [39], the authors propose two schemes, packet collision avoidance and Interest packet aggregation for emerging and tactical MANETs. In these schemes, two extra packets (e.g. Request and reply) in addition to conventional NDN packets are used, which cause extra network overhead. In a previous study, the authors [40] utilized the NDN approach in wireless networks to reduce the video packet loss. They also introduce a new timeout algorithm for the measurement of variations in route-trip time estimation.

Yu-Ting et al., in [41], elaborated upon a novel scheme (i.e., NAIF) to avoid the extra packet flooding in the network. In this scheme, a packet is forwarded or dropped based on the distance information and the data download rate. In a previous study, the authors [42] presented a scheme to download the content from mobile cloud. In this approach, the forwarder node is selected in each quadrant. Two extra packets (i.e., CMD, ACK) are also utilized in this process. Dabin et al., in [43], describe a broadcasting protocol for NDN-based wireless ad hoc networks.

In [45], two novel forwarding protocols, blind forwarding $(\mathrm{BF})$ and provider-aware forwarding (PAF), are presented by the authors. The BF protocol describes the controlled flooding mechanism and reduces the Interest and Data packet flooding in the network. On the other hand, the PAF protocol utilizes a distance-based forwarding approach that is based on the hop count. In addition, both protocols do not consider the remaining energy of the nodes during their communication process.

However, none of above mentioned schemes take the node's remaining energy concept into account in their forwarding process. Most previously proposed schemes utilize the leave copy everywhere (LCE) caching policy. As a result, the number of duplicate Data packets is also increased in the network.

\section{METHODLOGY}

\section{PROBLEM DESCRIPTION:}

Scenario of the NDN-based MANETs, in which mobile nodes can freely move anywhere. If a consumer node needs some content from a provider node, it follows the conventional NDN communication procedure and broadcasts an Interest packet to the provider node. Upon reception of the Interest packet, relay node 2 checks its CS for the availability of a Data packet. If it has data, relay node 2 sends it back to the consumer node. Otherwise, it further checks its PIT table. If there is no entry available, relay node 2 adds the Interest packet entry in the PIT table and further rebroadcasts the Interest packet toward other nodes. Similar to node 2, the other relay nodes (i.e., node 1 and node 4) adopt the same process of Interest packet forwarding. When node 2rebroadcasts the Interest packet, the provider node as well as node 5 and node 6 also receive the same Interest packet. Nodes 5 and 6 (which do not have Data packets) further rebroadcast the Interest packet to other nodes using the same process as discussed before. In addition, nodes 8, 9, and 10 also follow the same forwarding process. Hence, there is unnecessary Interest packet flooding in the network. 
Hence, there is unnecessary Interest packet flooding in the network. The numbers in parentheses in the figure represent the sequence numbers of both Interest and Data packets issued in the network. Upon reception of the Interest packet, the provider node replies with the Data packet. When the Data packet comes back toward the consumer node, it simply follows the pending requests entries previously stored in the PIT tables of the relay nodes. When the Data packet is received by relay node 2 , it checks its PIT table for corresponding entry. If there is an entry, the relay node 2 forwards the Data packet toward the consumer node. It also removes the entry from the PIT table. Due to the Interest packet flooding, the similar request entries are stored on multiple nodes. The numbers in parentheses in the figure represent the sequence numbers of both Interest and Data packets issued in the net-work. Upon reception of the Interest packet, the provider node replies with the Data packet. When the Data packet comes back toward the consumer node, it simply follows the pending requests entries previously stored in the PIT tables of the relay nodes. When the Data packet is received by relay node 2 ,it checks its PIT table for a corresponding entry. If there is an entry, the relay node 2 forwards the Data packet towards the consumer node. It also removes the entry from the PIT table. Due to the Interest packet flooding, the similar request entries are stored on multiple nodes.

\section{LOMCF Protocol Description}

We present a novel protocol, the LOMCF for NDN-based MANETs. The proposed LOMCF protocol considers the locations of both a consumer and a provider during its forwarding mechanism. A relay node forwards the Interest or Data packet only if it has less distance towards the consumer or provider node, respectively. This mechanism reduces the unnecessary packet flooding in the network. In addition, the forwarding process also considers the node's remaining energy to improve the performance of the nodes. The LOMCF protocol utilizes the multipath forwarding process that has two benefits: first, high reliability is accomplished for transmitting both Interest and Data packets. Second, Data packets follow multiple routes .As a result, content is widely distributed over the network, which mitigates the content retrieval time for future requests.

The LOMCF protocol also introduces a distancebased caching policy that takes into account the Data packet duplication in the network. According to this policy, it stores Data packets near the consumer compared to the provider node Moreover, the LOMCF protocol also mitigates the Interest and Data packet collisions probability using a timer-based forwarding process. In the LOMCF protocol, a relay node lies into two modes based on its location and remaining energy. A relay node is in a potential Interest forwarder (PIF) mode if

$$
D_{c p}<=D_{p p} \text { and } E_{r}>E_{t h}
$$

where Dpp and Dcp denote the distance from a previous relay node to a provider node and from a current relay node to a provider node, respectively. The distance is calculated using a well-known Euclidean distance formula as follows:

$$
D_{i j}=\sqrt{\left(x_{j}-x_{i}\right)^{2}+\left(y_{j}-y_{i}\right)^{2}}
$$

where the $\mathrm{xi}$ and $\mathrm{yi}$ represent the position coordinates of the respective node i. In Fig. 4, node 2 is closer to the provider node (Dcp) than the consumer node (Dpp). Moreover, if it has higher remaining energy, then it is considered in PIF mode. Er shows the remaining energy of the node, and Eth represents the energy threshold. As explained in Section 5 (A),after extensive 
simulations, the value of Eth is set to $13 \%$ for evaluating the proposed protocol. If $\mathrm{Er}<\mathrm{Eth}$, then the node is considered in the Critical State. When a node is in the Critical State, it does not further send the Interest packets .Instead, it focuses on Data packet transmissions and satisfies its PIT table's entries. A relay node is in a potential Data forwarder (PDF) mode and caches Data packets if

$$
D_{c c}<=D_{p c}
$$

where Dcc and Dpc denote the distance from a current relay node to a consumer node and from a previous relay node to a consumer node, respectively.

1) Packet Format and Node's Architecture:

The LOMCF protocol maintains the tenet of the conventional NDN and does not use any additional buffers, control packets, or data.

\section{TABLE I: INT-PAC format}

\begin{tabular}{c|l}
\hline Field & \multicolumn{1}{c}{ Deseription } \\
\hline INT-PAC Name & Requested content name \\
\hline Nonce & $\begin{array}{l}\text { Random value use to avoid the duplication of INT- } \\
\text { PAC }\end{array}$ \\
\hline INT-PAC Lifetime & $\begin{array}{l}\text { It shows the lifetime of the INT-PAC and specified } \\
\text { in seconds }\end{array}$ \\
\hline Conumser Position & $\begin{array}{l}\text { It contains the }(x, y) \text { coordinates of a consumer } \\
\text { node }\end{array}$ \\
\hline Relay Position & It contains the $(x, y)$ coordinates of a relay node \\
\hline Provider Position & It contains the $(x, y)$ coordinates of a provider node \\
\hline Options & $\begin{array}{l}\text { It contains the information regarding order prefer- } \\
\text { ences, excluded filters etc. }\end{array}$ \\
\hline
\end{tabular}

However, for the LOMCF protocol, the formats of both of Interest and Data packets are revised and the proposed protocol utilizes the two packets to implement the functionalities of LOMCF protocol. For simplicity, here in after, we call an Interest packet INT-PAC and a Data packet DATA PAC. In addition to the fields of packet formats of NDNs, the revised packets include three new fields: the consumer position, relay position, and provider position, as shown in Tables 1 and 2.

TABLE II: DATA-PAC format

\begin{tabular}{c|l}
\hline Field & \multicolumn{1}{c}{ Description } \\
\hline DATA-PAC Name & Requested content name \\
\hline DATA-PAC Length & Length of the requested content \\
\hline DATA-PAC Type & $\begin{array}{l}\text { It contains information about the content type, } \\
\text { such as text, audio, video etc. }\end{array}$ \\
\hline DATA-PAC Content & Actual data payload \\
\hline Conumser Position & $\begin{array}{l}\text { It contains the }(\mathrm{x}, \mathrm{y}) \text { coordinates of a consumer } \\
\text { node }\end{array}$ \\
\hline Relay Position & It contains the $(\mathrm{x}, \mathrm{y})$ coordinates of a relay node \\
\hline Provider Position & It contains the $(\mathrm{x}, \mathrm{y})$ coordinates of a provider node \\
\hline Signature Type & $\begin{array}{l}\text { That represents the type of signature, such as } \\
\text { SHA256, empty signature, SHA256 with RSA etc. }\end{array}$ \\
\hline
\end{tabular}

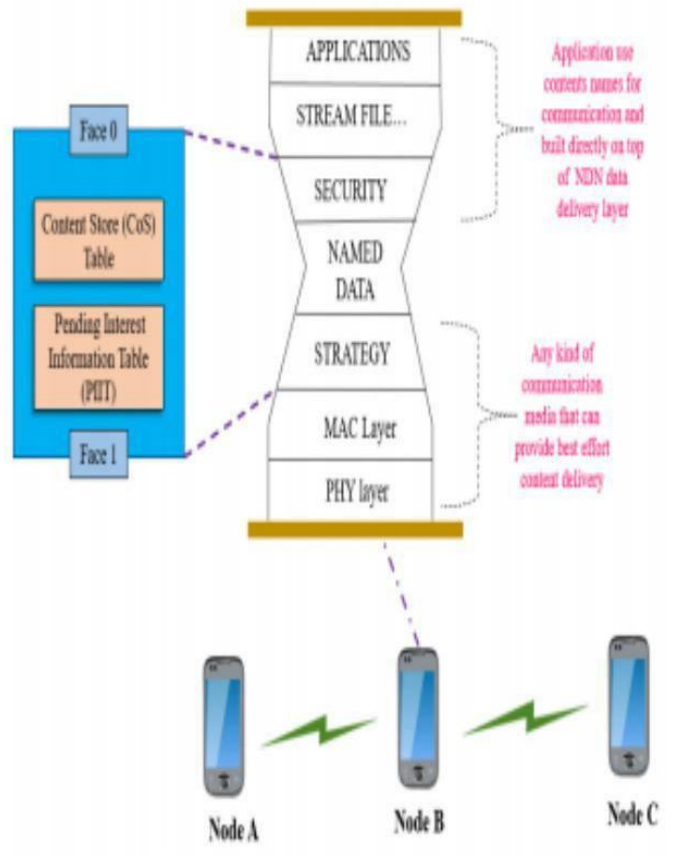

A basic LOMCF node's architecture is depicted in Fig. . In the LOMCF protocol, the participating nodes also prevent packet duplications and collisions. When a node receives an INT-PAC or DATA-PAC, it randomly defers and senses the channel for TINT -P AC or TDAT A-P AC times, respectively. TINT $-P$ AC and TDAT A-P AC values are calculated as follows [45]. TINT $-P$ AC $=\left(\right.$ Interval $+\operatorname{rand}[0, \text { Interval] })_{\text {Ineral-11) }}$ SLOT time.(4)

TDAT A-P AC $=($ rand $[0$, time (5) where Interval denotes the integer value that represents the length of the time interval and 
SLOT time denotes the fixed short time interval. Moreover, rand() represents a random function that generates the different random values in a specified range. If the identical transmissions (i.e., duplicate INT-PACs or DATA-PACs) are received during this time period, the node discards the current transmission. The following sections describe the content discovery and delivery processes of LOMCF protocol.

\section{Content Discovery Mechanism:}

When a consumer node requires content, it broadcasts an INT-PAC by including the content name and its current position (e.g., $\mathrm{x}$ and $\mathrm{y}$ coordinates) toward the neighbor nodes. Initially, the consumer node does not know the provider node's location; it simply sets the relay and provider position fields of INT-PAC to its current position and null value, respectively. The participating relay nodes simply rebroadcast the INT-PAC until the provider node is reached. Upon reception of an INT-PAC, the provider node replies with a DATA-PAC, which also includes its current location information (i.e., $\mathrm{x}, \mathrm{y}$ coordinates) in the provider position field of the DATA-PAC. Moreover, the provider node also includes the consumer location in the DATA-PAC's consumer field that is extracted from the INT-PAC. DATAPAC delivery is discussed in greater depth in section 4.B(3). After receiving the first DATAPAC, the consumer node extracts the provider location information from the DATA-PAC and includes this information in the subsequent INTPAC transmissions.

\section{Content Delivery Mechanism:}

When an INT-PAC reaches its destination, the provider node replies with the DATA-PAC toward the consumer node, which also includes the locations information of both provider and consumer nodes.

\section{MODULES:}

\section{Topology Creation:}

Create topology of a wireless ad hoc networks by a graph $\mathrm{G}(\mathrm{V} ; \mathrm{E})$, where $\mathrm{V}$ and $\mathrm{E}$ are the set of nodes (vertices) and links (edges), respectively. Each node is assigned a unique integer identifier between 1 and $\mathrm{N}=|\mathrm{V}|$. Nodes are assumed to be battery powered. The remaining battery energy of node $u$

\section{$\mathrm{EV}$ is represented by $\mathrm{Cu}$. If the battery energy of a}

node falls below a threshold $\mathrm{Cth}$, the node is considered to be dead. Without loss of generality, we assume $\mathrm{Cth}=0$. A link in the network is denoted by $(\mathrm{u} ; \mathrm{v})$, in which $\mathrm{u}$ and $\mathrm{v}$ are sending and receiving nodes, respectively. The criterion for having a link from $\mathrm{u}$ to $\mathrm{v}$ is as follows: There could be a link from $\mathrm{u}$ to $\mathrm{v}$, if the received signal strength by $\mathrm{v}$ is above a threshold. This threshold is usually specified in such a way that a targeted link error probability is satisfied.

\section{Find Possible Path:}

The possible paths between a source and a destination node are found using Djikisthra's algorithm. The sender can select a path and send message to destination.

\section{Message Sending with caching:}

When sender or neighbor sends message, a copy of message is saved in cache. This message is used for retransmission purpose, if acknowledgement not received from receiver.

\section{Message Retransmission:}

The node waits for ACK from receiver or neighbor. If $\mathrm{ACK}$ is not received within timeout period, the message will be retransmitted. This process is done until ACK is received from receiver. 


\section{Message Receiver:}

Receiver sends ACK for every received message. If receiver does not send ACK, message is retransmitted from neighbor until ACK is received.

\section{ACKNOWLEDGEMENT}

We wish to offer our sincere gratitude to our principal Dr. Prof .Channakeshvalu for providing me an opportunity to be a part of the East West Institute of Technology, Bangalore under the graduate program and moreover to allow me work on this final year project on the respective domain.

We would like to offer gratitude to the H.O.D of Computer Science and Engg, East West Institute of Technology ,Dr. Arun Biradar for his continuous support and encouragement towards our project.

We sincerely thank our guide Prof. Prasanna Kumar , for the guidance and encouragement in carrying out this work and rectifying the mistakes in the same for the betterment of our work.

\section{REFERENCES}

[1] T. Anderson, L. Peterson, S. Shenker, and J. Turner, "Overcoming the internet impasse through virtualization,” Computer, no. 4, pp. 34-41, 2005. https://doi.org/10.1109/MC.2005.136

[2] "Cisco visual networking index: forecast and methodology: 2011-2015,” May 2012.

[3] "Google, we knew the web was big." http://googleblog.blogspot.com/2008/07/we-knewweb-was-big.html.

[4] J. Lee and D. Kim, "Proxy-assisted content sharing using content centric networking (ccn) for resource-limited mobile consumer devices," Consumer Electronics, IEEE Transactions on, vol. 57, no. 2, pp. 477-483, 2011.

https://doi.org/10.1109/TCE.2011.5955182

[5] A. Passarella, "A survey on content-centric technologies for the current internet: $\mathrm{Cdn}$ and $\mathrm{p} 2 \mathrm{p}$ solutions," Computer Communications, vol. 35, no. 1, pp. 1-32, 2012.

https://doi.org/10.1016/j.comcom.2011.10.005

[6] B. Ahlgren, C. Dannewitz, C. Imbrenda, D. Kutscher, and B. Ohlman, "A survey of information-centric networking," Communications Magazine, IEEE, vol. 50, no. 7, pp. 26-36, 2012. https://doi.org/10.1109/MCOM.2012.6231276
[7] A. Detti, D. Tassetto, N. B. Melazzi, and F. Fedi, "Exploiting content centric networking to develop topic-based, publish-subscribe manet systems," Ad Hoc Networks, vol. 24, pp. 115-133, 2015.

https://doi.org/10.1016/j.adhoc.2014.07.020

[8] "Pursuit: Pursuing a pub/sub internet. http://www.fp7-pursuit.eu/."

[9] M. Brunner, H. Abramowicz, N. Niebert, and L. M. Correia, "4ward: a european perspective towards the future internet," IEICE transactions on communications, vol. 93, no. 3, pp. 442-445, 2010. https://doi.org/10.1587/transcom.E93.B.442

[10] "Comet: Content mediator architecture for content-aware networks. http://www.cometproject.org/."

[11] V. Jacobson, D. K. Smetters, J. D. Thornton, M. F. Plass, N. H. Briggs, and R. L. Braynard, "Networking named content," in Proceedings of the 5th international conference on Emerging networking experiments and technologies. ACM, 2009, pp. 1-12. https://doi.org/10.1145/1658939.1658941

[12] "Convergence: Host centric to contentcentric. http://www.ictconvergence.eu/."

[13] "Coast: Content aware searching retrieval and streaming. http://www.coast-fp7.eu/." [14] "The greenicn project, http://www.greenicn.org/.", 